\title{
The predictors of sustained virological response with sofosbuvir and ribavirin in patients with chronic hepatitis $C$ genotype 2
}

Sung Yong $\mathrm{Han}^{1,2}$, Hyun Young Woo ${ }^{1,2}$, Jeong Heo ${ }^{1,2}$, Sang Gyu Park ${ }^{1,2}$, Sung Ik Pyeon ${ }^{1,2}$, Young Joo Park ${ }^{1,2}$, Dong Uk Kim ${ }^{1,2}$, Gwang Ha Kim ${ }^{1,2}$, Hyung Hoi Kim ${ }^{2,3}$, Geun Am Song, ${ }^{1,2}$, and Mong Cho ${ }^{1,2}$

${ }^{1}$ Department of Internal Medicine, Pusan National University School of Medicine, Busan; ${ }^{2}$ Medical Research Institute, Pusan National University Hospital, Busan; ${ }^{3}$ Department of Laboratory Medicine, Pusan National University School of Medicine Busan, Korea

Received: September 9, 2018 Revised : December 15, 2018 Accepted: January 23, 2019

\section{Correspondence to}

Jeong Heo, M.D.

Department of Internal Medicine, Pusan National University School of Medicine and Medical Research Institute, Pusan National University Hospital, 179 Gudeok-ro, Seo-gu, Busan 49241, Korea

Tel.: +82-51-240-7869

Fax: +82-51-244-8180

E-mail:jheo@pusan.ac.kr

\section{Hyun Young Woo, M.D.}

Department of Internal Medicine, Pusan National University School of Medicine and Medical Research Institute, Pusan National University Hospital, 179 Gudeok-ro, Seo-gu, Busan 49241, Korea

Tel: +82-51-240-7869

Fax: +82-51-254-3237

E-mail: who54@hanmail.net

Background/Aims: Real-world, clinical practice data are lacking about sofosbuvir/ ribavirin (SOF/RBV) treatment of Korean patients with hepatitis $\mathrm{C}$ virus genotype 2 (HCV GT2) infection. This study investigated the efficacy and safety of SOF/RBV in Korean patients with HCV GT2 infection and clinical factors predicting sustained virological response 12 weeks (SVR12) after the end of SOF/RBV treatment. Methods: A total of 181 patients with HCV GT2 with/without cirrhosis were treated with SOF/RBV for 16/12 weeks. Rapid virological response (RVR) was defined as non-detectable HCV RNA at 4 weeks.

Results: The RVR rate was $80.7 \%$ (146/181), the end of treatment response rate was $97.8 \%$ (177/181) and the SVR12 rate was 92.8\% (168/181). Of eight patients with relapse, four did not achieve RVR. Three patients had a history of hepatocellular carcinoma (HCC). Multivariable analysis showed that RVR $(p=0.015)$ and no previous history of HCC $(p=0.007)$ were associated with SVR12. Factors significantly contributing to RVR included cirrhosis, creatinine concentration, and pre-treatment HCV RNA level. SVR12 rate was significantly higher in RVR (+) than RVR (-) patients $(95.2 \%$ vs. $82.9 \%, p=0.011)$ and also significantly higher in patients without than with a history of HCC (94.1\% vs. $72.7 \%, p=0.008)$. During treatment, $80 / 181$ patients (44.2\%) experienced mild to moderate adverse events, with 32 (17.7\%) requiring RBV dose reductions due to anemia.

Conclusions: SOF/RBV treatment was effective and tolerable in HCV GT2 patients. RVR and no previous history of HCC were positive predictors of SVR12.

Keywords: Hepatitis C, chronic; Sustained virological response; Rapid virological response; Sofosbuvir; Carcinoma, hepatocellular

\section{INTRODUCTION}

Approximately 8o million people worldwide are chronically infected with hepatitis C virus (HCV) [1]. Chronic hepatitis $\mathrm{C}(\mathrm{CHC})$ is a major cause of end stage liver disease and hepatocellular carci- noma (HCC) $[2,3]$. Of the seven HCV genotypes (GTs) identified, HCV genotype 2 (GT2) is the third most common, affecting an estimated 16.5 million persons worldwide. In East Asia, around $20 \%$ to $45 \%$ of patients with CHC are infected with HCV GT2 [1,4-8]. 
Sustained virological response 12 weeks after the end of treatment (SVR12) is associated with reduced risks of liver-related morbidity and mortality [9,10]. Treatment with pegylated interferon (PegIFN) and ribavirin (RBV) for 24 weeks has yielded higher response rates in patients infected with HCV GT2 than in patients infected with other HCV GTs, such as GT1, GT3, and GT4. However, the poor tolerability associated with PegIFN-based regimens has limited their clinical use, especially in patients with liver cirrhosis (LC).

All-oral, direct-acting antiviral (DAA) drug combinations have shown high efficacy rates, favorable side-effect profiles and easy applicability in treatment of HCV infection. The combination of sofosbuvir (SOF) and RBV was the first all-oral regimen recommended by the American Association for the Study of Liver Diseases (AASLD) [11] and approved by national health care services in Asian countries for the treatment of patients infected with HCV GT2. However, real-world, clinical practice data are lacking about SOF/RBV treatment of Korean patients with HCV GT2 infection.

Factors affecting SVR12 in patients treated with DAAs include $\mathrm{LC}, \mathrm{GT}_{3}$, previous treatment failure, and resistance-related mutations [12]. AASLD guidelines recommend extended treatment, for up to 16 weeks, in HCV-infected patients with LC [11]. However, predictors of SVR12 have not been well investigated in real clinical practice. The present study therefore investigated the efficacy and safety of SOF/RBV in Korean patients with HCV GT2 infection, as well as clinical factors predictive of SVR12 in these patients.

\section{METHODS}

\section{Patients}

This retrospective observational cohort study included patients with HCV GT2 who were treated with SOF/RBV at Pusan National University Hospital between May 2016 and December 2017. Patients not treated for at least 12 weeks were excluded. CHC was diagnosed by laboratory and/or serologic tests, and HCV GT2 was identified by real time-polymerase chain reaction (RT-PCR) and hybridization. Patients were treated according to Korean National Health Insurance guidelines. Patients who were not treated with or failed previous PegIFN-based regimens, and those not treated with other HCV protease inhibitors, were included, whereas patients who had undergone liver transplantation were excluded. This study was performed in accordance with the ethical guidelines of the Helsinki Declaration (revised in 2013), and the study protocol was approved by the Institutional Review Board of Pusan National University (IRB No. H-1803-010-064). All patients provided written informed consent before starting treatment.

\section{Treatment protocol}

Patients were administered $400 \mathrm{mg} /$ day SOF (SOVALDI, Gilead Sciences, Foster City, CA, USA) plus weight-adjusted RBV (VIRAMID, Ilsung Pharm., Seoul, Korea), with patients weighing $<75 \mathrm{~kg}$ administered 1,000 mg/day RBV and those weighing $\geq 75 \mathrm{~kg}$ administered $1,200 \mathrm{mg} /$ day RBV. Patients without cirrhosis were treated for 12 weeks and patients with cirrhosis for 16 weeks. RBV doses were adjusted for complications, including reduced hemoglobin concentrations. RBV dose was reduced by $200 \mathrm{mg} /$ day if a patient's hemoglobin level was below 10 $\mathrm{mg} / \mathrm{dL}$; if hemoglobin level subsequently returned to the normal range for 4 weeks, RBV dose was increased by $200 \mathrm{mg} /$ day [13]. RBV was discontinued in patients with hemoglobin levels below $8.5 \mathrm{mg} / \mathrm{dL}$ [13]. Laboratory tests, including HCV RNA titers, complete blood counts, and liver and renal function tests, were performed before treatment, after 4 weeks, at the end of treatment, and 12 weeks after the end of treatment. Pre-treatment estimated glomerular filtration rate (eGFR) was calculated as 175 $\times$ serum creatinine $e^{(-1.154)} \times$ age $e^{(-0.203)}(\times 0.742$, if female $)$. Serum RNA levels were measured by RT-PCR (COBAS TaqMan Analyzer, Roche Molecular Systems Inc., Pleasanton, CA, USA), with a lower quantitative detection limit of $15 \mathrm{IU} / \mathrm{mL}$; non-detection and concentrations below $15 \mathrm{IU} / \mathrm{mL}$ were reported separately.

Cirrhosis was defined as the presence of at least two of the following characteristics: platelet count $<140,000 /$ $\mathrm{mL}$, evidence of esophageal varices on esophagogastroduodenoscopy, cirrhosis and/or portal hypertension and/or ascites on imaging modalities, and fibroscan results compatible with stage 4 fibrosis. Decompensated cirrhosis was defined as current or previous presence of ascites, hepatic encephalopathy, spontaneous bacterial peritonitis, hepatic hydrothorax, variceal hemorrhage, or concomitant treatment with medications specifically 
Table 1. Baseline demographic and clinical characteristics of all patients $(n=181)$

\begin{tabular}{lc}
\hline Variable & Value \\
\hline Male sex & $76(42.0)$ \\
\hline Age, yr & $61.4 \pm 11.5$ \\
\hline Weight, kg & $62.7 \pm 11.1$ \\
\hline Liver cirrhosis & $50(27.6)$ \\
\hline History of HCC & $11(6.1)$ \\
\hline Diabetes & $30(16.6)$ \\
\hline Treatment naive & $143(79.0)$ \\
\hline Laboratory findings & \\
\hline White blood cell count, / $\mathrm{L}$ & $5,595.4 \pm 1,796.5$ \\
\hline Hemoglobin, g/dL & $13.3 \pm 1.6$ \\
\hline Platelets, 10 $3 / \mathrm{uL}$ & $177.4 \pm 71.7$ \\
\hline Aspartate transaminase, IU/L & $46.3 \pm 35.0$ \\
\hline Alanine aminotransferase, IU/L & $36.2 \pm 31.7$ \\
\hline Albumin, g/dL & $4.1 \pm 0.4$ \\
\hline Total bilirubin, mg/dL & $0.73 \pm 0.39$ \\
\hline Creatinine, mg/dL & $0.75 \pm 0.19$ \\
\hline eGFR, mL/min/1.73 m ${ }^{2}$ & $96.1 \pm 23.2$ \\
\hline Baseline HCV RNA, IU/mL & $4,453,841 \pm 12,806,387$ \\
\hline
\end{tabular}

Values are presented as number (\%) or mean $\pm \mathrm{SD}$. HCC, hepatocellular carcinoma; eGFR, estimated glomerular filtration rate; $\mathrm{HCV}$, hepatitis $\mathrm{C}$ virus.

indicated for the above-mentioned conditions.

\section{Definition of response}

Rapid virological response (RVR) was defined as non-detection of HCV RNA 4 weeks after starting treatment. HCV RNA titer under the lower quantitative limit (< $15 \mathrm{IU} / \mathrm{mL}$ ) was defined as not attaining RVR. End of treatment response (ETR) and SVR12 were defined as non-detection of HCV RNA at the end of treatment and 12 weeks after the end of treatment, respectively. Adverse events (AEs) were recorded regularly in patients' medical records. Anemia was defined as hemoglobin concentration $<10 \mathrm{~g} / \mathrm{dL}$ or a $\geq 2.0 \mathrm{~g} / \mathrm{dL}$ decrease in hemoglobin concentration. RBV dose modifications were also documented

\section{Statistical analysis}

All statistical analyses were performed using IBM SPSS statistical software version 21.0 (IBM Co., Armonk, NY,
USA). All data were calculated in the intention to treat (ITT) population, defined as all patients who received at least one tablet of SOF/RBV. Continuous variables were expressed as mean and standard deviation and compared by independent sample $t$ tests. Categorical variables were expressed as absolute numbers and relative frequencies, with qualitative and quantitative variables compared using the chi-square test and the Mann-Whitney $U$ test, respectively. Logistic regression analysis was used to identify factors predicting SVR12. Factors with $p$ values $<0.2$ on univariate analysis were included in multivariate analysis, along with clinically meaningful variables. A two sided $p$ value $<0.05$ was considered statistically significant.

\section{RESULTS}

\section{Patient characteristics}

During the study period, 408 patients were diagnosed with HCV GT2. Of these, 218 were not treated with SOF/ RBV, including 143 with current HCC, 19 with eGFR $<30 \mathrm{~mL} / \mathrm{min} / 1.73 \mathrm{~m}^{2}$ and 56 for economic reasons. Of the remaining 190 patients who started SOF/RBV treatment, nine were excluded because they had previously undergone liver transplantation. Thus, 181 patients were included in this study; their baseline demographic and clinical characteristics are shown in Table 1 . The 181 patients included 105 women (58.0\%) and 76 men (42.0\%), of mean age $61.4 \pm 11.5$ years. Of these 181 patients, 143 (79.0\%) were treatment naïve, 11 (6.1\%) had a history of HCC, 30 (16.6\%) had diabetes mellitus, and 50 (27.6\%) had cirrhosis, including six with decompensation. Their mean pre-treatment serum HCV RNA titer was $4.45 \pm$ $12.80 \times 10^{6} \mathrm{IU} / \mathrm{mL}$, their mean hemoglobin concentration was $13.3 \pm 1.6 \mathrm{~g} / \mathrm{dL}$, their mean body weight was 62.7 $\pm 11.1 \mathrm{~kg}$ and their mean serum creatinine concentration was $0.75 \pm 0.19 \mathrm{mg} / \mathrm{dL}$. Fig. 1 shows the flow chart of the study. We intended to treat 50 patients with cirrhosis for 16 weeks; however, two of these patients experienced severe diarrhea and were treated for only 12 weeks. Therefore, 48 patients with cirrhosis were treated for 16 weeks and 131 patients without cirrhosis and two with cirrhosis were treated for 12 weeks. 


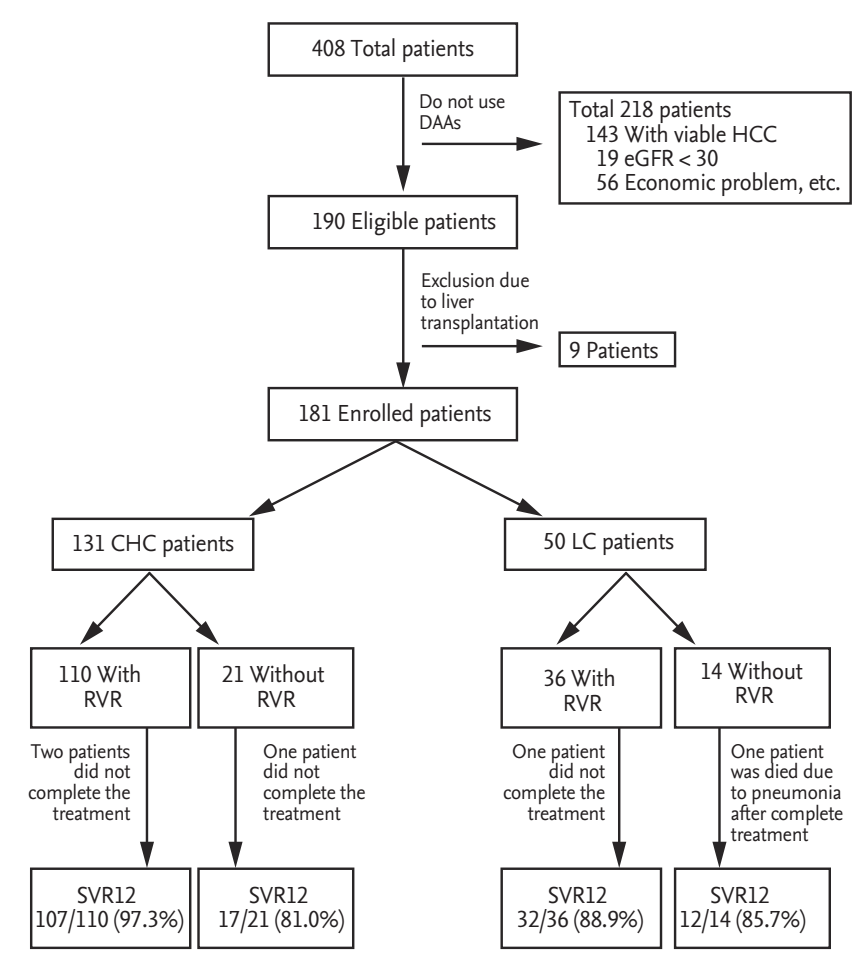

Figure 1. Study flow chart. DAA, direct-acting antiviral; HCC, hepatocellular carcinoma; eGFR, estimated glomerular filtration rate; CHC, chronic hepatitis C; LC, liver cirrhosis; RVR, rapid virological response; SVR, sustained virological response.

\section{Treatment response}

Of the 181 patients who started SOF/RBV treatment, four did not complete treatment and one was lost to post-treatment follow-up. ITT analysis showed an RVR rate of $80.7 \%$ (146/181), an ET'R rate of $97.8 \%$ (177/181) and an SVR12 rate of $92.8 \%$ (168/181). SVR12 rates were $88.0 \%$ (44/50) in patients with cirrhosis and $94.7 \%$ (124/131) in patients without cirrhosis. SVR12 rates were 83.3\% (5/6) in patients with decompensated LC and 88.9\% (39/44) in patients with compensated LC, with no significant between-group difference $(p=0.708)$. One patient with decompensated LC who did not achieve SVR12 was lost to follow-up, with no evidence of actual HCV recurrence. Of the 133 patients treated for 12 weeks, 126 (94.7\%) achieved SVR12, including both (100\%) patients with cirrhosis and 124 (94.7\%) of 131 without cirrhosis. Of the 48 patients treated for 16 weeks, all with cirrhosis, 42 (87.5\%), achieved SVR12. Table 2 shows clinical information on patients who showed recurrence of $\mathrm{HCV}$. Of the

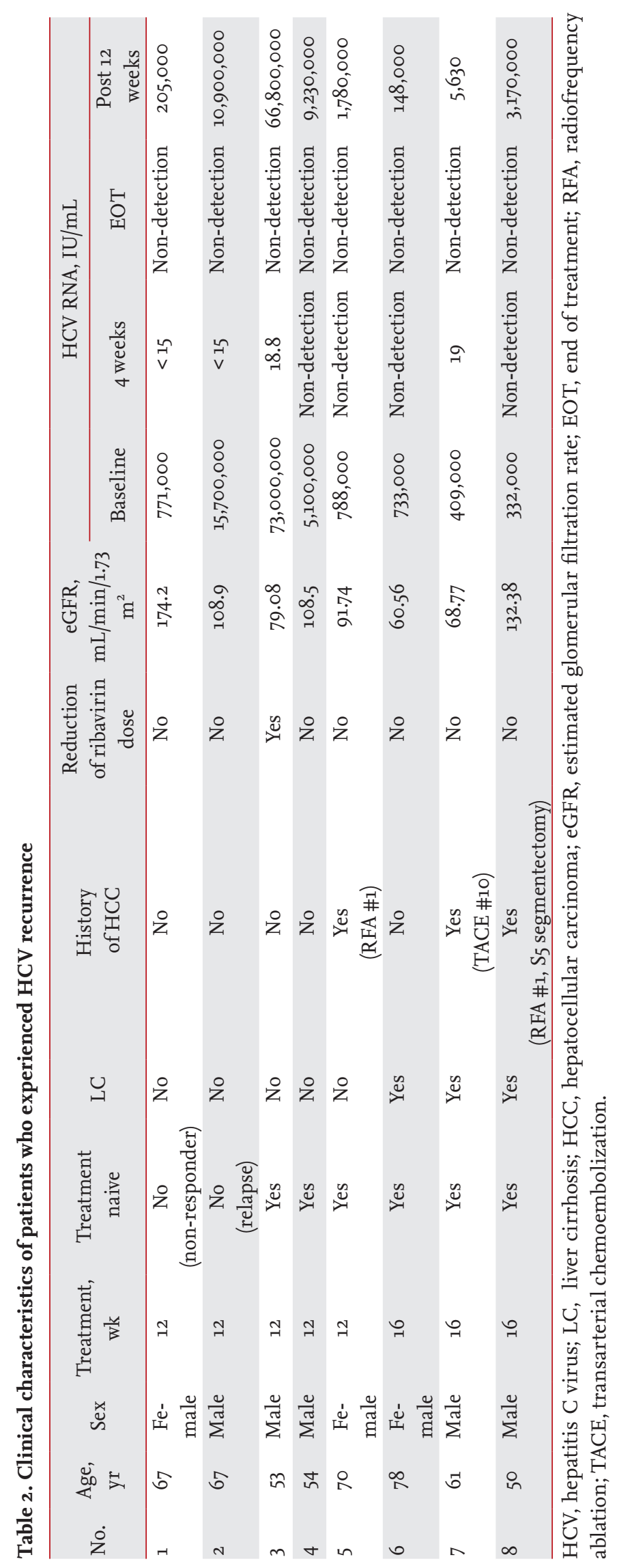


Table 3. Clinical factors associated with sustained virological response at 12 weeks

\begin{tabular}{|c|c|c|c|c|}
\hline \multirow{2}{*}{ Variable } & \multirow{2}{*}{ Patients without SVR12 } & \multirow{2}{*}{ Patients with SVR12 } & \multicolumn{2}{|c|}{$p$ value } \\
\hline & & & Univariate & Multivariate \\
\hline Total patients & $13 / 181(7 \cdot 2)$ & 168/181 (92.8) & & \\
\hline Male sex & $7 / 76(9.2)$ & $69 / 76(90.8)$ & 0.270 & 0.124 \\
\hline Age, yr & $67(50-84)$ & $60(43-86)$ & 0.193 & \\
\hline$>60$ & $9 / 86(10.5)$ & $77 / 86(89.5)$ & 0.104 & 0.711 \\
\hline Weight, kg & $63.0(49-90.9)$ & $60(35-104)$ & 0.530 & \\
\hline$>60$ & $8 / 92(8.7)$ & $84 / 92(91.3)$ & 0.423 & \\
\hline Liver cirrhosis & $6 / 50(12)$ & $44 / 50(88)$ & 0.112 & 0.619 \\
\hline Decompensated & $1 / 6(16.7)$ & $5 / 6(83.3)$ & 0.708 & \\
\hline Diabetes & $2 / 30(6.7)$ & $28 / 30(93 \cdot 3)$ & 0.632 & \\
\hline Treatment naive & $11 / 143(7 \cdot 7)$ & $132 / 143(92.3)$ & 0.460 & 0.824 \\
\hline History of HCC & $3 / 11(27 \cdot 3)$ & $8 / 11(72.7)$ & 0.034 & 0.007 \\
\hline 16 Weeks of treatment & $4 / 46(8.7)$ & $42 / 46(91.3)$ & 0.195 & 0.697 \\
\hline Reduction of ribavirin dose & $2 / 32(6.2)$ & $30 / 32(93.8)$ & 0.588 & 0.787 \\
\hline \multicolumn{5}{|l|}{ Laboratory findings } \\
\hline White blood cell count, / $\mu \mathrm{L}$ & $5,130(2,910-9,530)$ & $5,300(2,270-11,430)$ & 0.929 & \\
\hline$>5,280$ & $6 / 91(6.6)$ & $85 / 91(93.4)$ & $0.75^{8}$ & \\
\hline Hemoglobin, g/dL & $13.2(10.7-16.5)$ & $13.3(9.5-17.7)$ & 0.611 & \\
\hline$>13 \cdot 3$ & $5 / 90(5.6)$ & $85 / 90(94.4)$ & 0.399 & \\
\hline Platelets, $10^{3} / \mu \mathrm{L}$ & $160(48-329)$ & $179(23-425)$ & 0.567 & \\
\hline$>179$ & $6 / 89(6.7)$ & $83 / 89(83 \cdot 3)$ & 0.821 & \\
\hline Aspartate transaminase, IU/L & $40(18-115)$ & $35 \cdot 5(12-284)$ & 0.655 & \\
\hline$>36$ & $8 / 89(9)$ & $81 / 89(91.0)$ & 0.355 & \\
\hline Alanine aminotransferase, IU/L & $28(12-80)$ & $24 \cdot 5(8-264)$ & 0.573 & \\
\hline$>25$ & $7 / 85(8.2)$ & $78 / 85(91.8)$ & 0.606 & \\
\hline Albumin, g/dL & $4.16(2.5-4.7)$ & $4.23(2.5-4.9)$ & 0.138 & 0.954 \\
\hline$>4.2$ & $5 / 86(5.8)$ & $81 / 86(94.2)$ & 0.498 & \\
\hline Total bilirubin, mg/dL & $0.62(0.37-1.09)$ & $0.62(0.2-2.52)$ & 0.427 & \\
\hline$>0.62$ & $6 / 89(6.7)$ & $83 / 89(93 \cdot 3)$ & 0.821 & \\
\hline Creatinine, mg/dL & $0.76(0.37-1.09)$ & $0.71(0.38-1.71)$ & 0.393 & \\
\hline$>0.72$ & $8 / 90(8.9)$ & $82 / 90(91.1)$ & 0.377 & \\
\hline $\mathrm{eGFR}, \mathrm{mL} / \mathrm{min} / 1.73 \mathrm{~m}^{2}$ & $91.7(48.3-174.2)$ & $93.7(32.6-172.7)$ & 0.919 & \\
\hline$>93.5$ & $6 / 90(6.7)$ & $84 / 90(93 \cdot 3)$ & 0.773 & \\
\hline Baseline HCV RNA, $10^{3} \mathrm{IU} / \mathrm{mL}$ & $788(533-73,000)$ & $885(0.24-135,000)$ & 0.196 & 0.240 \\
\hline$>881$ & $6 / 90(6.7)$ & $84 / 90(93.3)$ & 0.789 & \\
\hline Rapid virological response & $6 / 145(4.8)$ & $139 / 145(95.2)$ & 0.021 & 0.015 \\
\hline
\end{tabular}

Values are presented as number/total number (\%) or median (range).

SVR, sustained virological response; HCC, hepatocellular carcinoma; eGFR, estimated glomerular filtration rate; HCV, hepatitis $\mathrm{C}$ virus. 


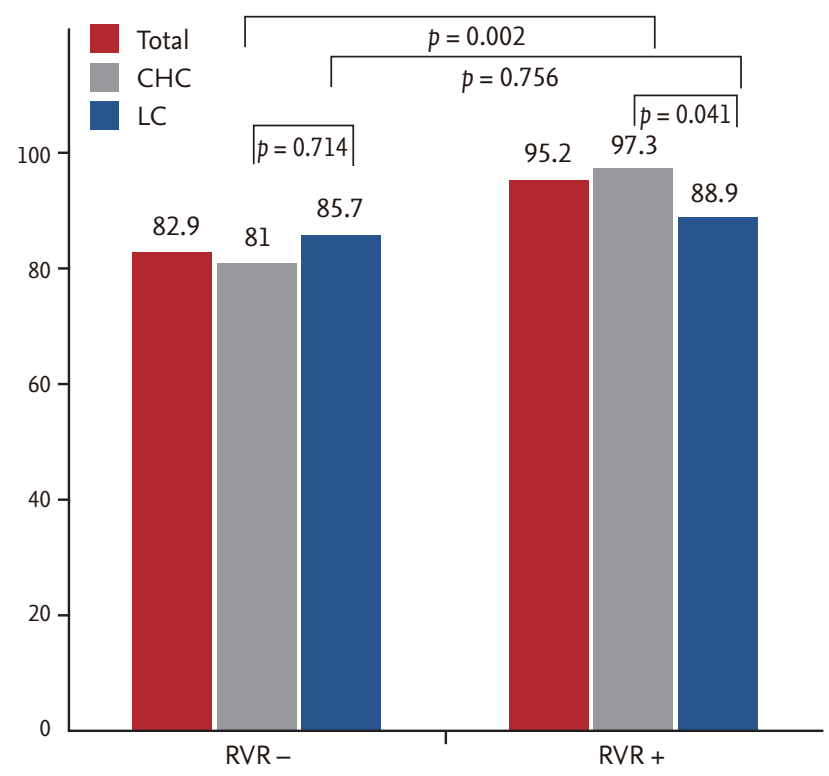

Figure 2. Rate of sustained virological response 12 weeks (SVR12) according to rapid virological response (RVR) in patients with and without liver cirrhosis. Among patients without liver cirrhosis (LC), SVR12 rate was significantly higher in those who did than did not achieve RVR (97.3\% vs. $81.8 \%, p=0.003)$. Among patients who achieved RVR, SVR 12 rate was significantly higher in patients without LC than with LC (97.3\% vs. 88.6\%, p=0.035). However, SVR12 was not significantly associated with RVR in patients with LC (84.6\% vs. $88.6 \%, p=0.720)$. CHC, chronic hepatitis C.

eight patients who relapsed, four did not achieve RVR, including two previously treated for HCV (one with relapse and one non-responder), and three patients with a history of HCC.

\section{Clinical factors associated with SVR12}

Table 3 shows clinical factors associated with SVR12. In univariate analysis, RVR $(p=0.021)$ and no previous history of $\mathrm{HCC}(p=0.034)$ were significantly associated with SVR12. Multivariate analysis showed that RVR $(p=0.015)$ and no previous history of HCC $(p=0.007)$ were significantly associated with SVR12.

Of the 181 patients, $146(80.7 \%)$ achieved RVR and 35 (19.3\%) did not. SVR12 rates in patients who did and did not achieve RVR were 95.2\% (139/146) and 82.9\% (29/35), respectively $(p=0.011)$. Fig. 2 shows the correlation of RVR and SVR12 according to LC status. SVR12 rates in patients with LC did not differ significantly in those with and without RVR (88.9\% vs. $85.7 \%, p=0.756)$. SVR12 rates in patients without $\mathrm{LC}$, however, were significantly higher in those who did than did not achieve RVR. (97.3\% vs. 81.0\%, $p=0.002$ ). SVR12 rates in patients who achieved RVR were significantly higher in those without than with LC (97.3\% vs. 88.9\%, $p=0.041)$. However, SVR12 rates among patients who did not achieve RVR were similar in those without and with LC $(81.0 \%$ vs. $85.7 \%, p=0.714$ ) (Table 4). Table 5 shows baseline factors associated with RVR. In univariate analysis, serum creatinine concentration $(p=0.012)$, previous treatment $(p$ $=0.031$ ), eGFR (Modification of Diet in Renal Disease; $p=0.016)$, and pre-treatment serum HCV RNA titer ( $p$ $=0.004)$ were significantly associated with RVR Multivariable analysis showed that $\mathrm{LC}(p=0.026)$, creatinine concentration $(p=0.015)$, and pre-treatment serum HCV RNA titer $(p=0.027)$ were significant predictors of RVR.

Of the 170 patients without and the 11 with a previous history of HCC, 160 (94.1\%) and eight (72.7\%), respectively, achieved SVR12 $(p=0.008)$. Mean CTP scores in these two groups were $5.4 \pm 0.9$ and $5.9 \pm 1.3$, respectively, a difference that was not statistically significant $(p=0.179)$. None of the patients in the study had CT'P class C. Of the 11 patients with HCC and the 40 with LCC but without HCC, one (9.1\%) and five (12.5\%), respectively, had CTP class B (Supplementary Table 1 ).

Table 6 shows the baseline characteristics associated with SVR12 in patients with a history of HCC. Univariate analysis showed that body weight, white blood cell count, and concentrations of aspartate transaminase and $\alpha$-fetoprotein were significantly associated with SVR12; whereas multivariate analysis showed that body weight and aspartate transaminase and $\alpha$-fetoprotein concentrations were significantly associated with SVR12. Before HCV treatment, all patients with HCC had achieved complete remission or had no viable portion of HCC following previous HCC treatment, including resection in two patients, radiofrequency ablation in five, and transarterial chemoembolization in four. After the end of HCV treatment, six patients, including four who achieved SVR12, showed HCC recurrence, with the median follow-up time from the end of HCV treatment to HCC recurrence being 6.5 months (range, 1.6 to 15.6).

\section{Adverse events}

During treatment, 80 (44.2\%) of the 181 patients experienced mild to moderate AEs (Table 7). RBV doses were 
Table 4. Clinical factors associated with sustained virological response at 12 weeks in patients without rapid virological response

\begin{tabular}{|c|c|c|c|c|}
\hline \multirow{2}{*}{ Variable } & \multirow{2}{*}{ Patients without SVR12 } & \multirow{2}{*}{ Patients with SVR12 } & \multicolumn{2}{|c|}{$p$ value } \\
\hline & & & Univariate & Multivariate \\
\hline Total patients & $6 / 35(17.1)$ & $29 / 35(82.9)$ & & \\
\hline Male sex & $4 / 13(30.8)$ & $9 / 13(69.2)$ & 0.120 & 0.091 \\
\hline Age, yr & $67(53-75)$ & $62(48-77)$ & 0.378 & \\
\hline$>60$ & $5 / 20(25)$ & $15 / 20(75)$ & 0.167 & 0.332 \\
\hline Weight, kg & $56.9(49.0-90.9)$ & $58.1(45.7-93.0)$ & 0.960 & \\
\hline$>60$ & $3 / 15(20)$ & $12 / 15(80)$ & 0.519 & \\
\hline Liver cirrhosis & $2 / 14(14 \cdot 3)$ & $12 / 14(85.7)$ & 0.544 & 0.506 \\
\hline Decompensated & $\mathrm{o} / \mathrm{o}(\mathrm{o})$ & o/o (o) & NA & \\
\hline Diabetes & $1 / 3(33 \cdot 3)$ & $2 / 3(66.7)$ & 0.442 & \\
\hline Treatment naive & $2 / 12(16.7)$ & $10 / 12(83 \cdot 3)$ & 0.671 & 0.658 \\
\hline History of HCC & $1 / 3(33 \cdot 3)$ & $2 / 3(66.7)$ & 0.442 & 0.615 \\
\hline 16 Weeks of treatment & $2 / 13(15 \cdot 4)$ & $11 / 13(84 \cdot 6)$ & 0.083 & 0.573 \\
\hline Reduction of ribavirin dose & $2 / 4(50.0)$ & $2 / 4(50.0)$ & 0.128 & 0.061 \\
\hline \multicolumn{5}{|l|}{ Laboratory findings } \\
\hline White blood cell count, $/ \mu \mathrm{L}$ & $5,965(2,910-9,530)$ & $5,530(2,510-8,820)$ & 0.694 & \\
\hline$>5,280$ & $3 / 21(14 \cdot 3)$ & $18 / 21(85.7)$ & 0.456 & \\
\hline Hemoglobin, g/dL & $13.3(10.7-16.5)$ & $13.3(9.9-17.1)$ & 0.521 & \\
\hline$>13 \cdot 3$ & $2 / 16(12.5)$ & $14 / 16(87.5)$ & 0.418 & \\
\hline Platelets, $10^{3} / \mu \mathrm{L}$ & $177(48-329)$ & $172(46-318)$ & $0.55^{2}$ & \\
\hline$>179$ & $3 / 16(18.8)$ & $13 / 16(81.3)$ & 0.582 & \\
\hline Aspartate transaminase, IU/L & $39 \cdot 5(18-115)$ & $32(14-201)$ & 0.733 & \\
\hline$>36$ & $4 / 18(22.2)$ & $14 / 18(77.8)$ & 0.358 & \\
\hline Alanine aminotransferase, IU/L & $15(12-30)$ & $23(8-110)$ & 0.170 & 0.177 \\
\hline$>25$ & $2 / 14(14 \cdot 3)$ & $12 / 14(85.7)$ & 0.544 & \\
\hline Albumin, g/dL & $4.15(2.5-4.3)$ & $4.1(3.30-4.80)$ & 0.187 & 0.198 \\
\hline$>4.2$ & $3 / 13(23.1)$ & $10 / 13(76.9)$ & 0.392 & \\
\hline Total bilirubin, mg/dL & $0.60(0.50-2.1)$ & $0.69(0.20-1.71)$ & 0.358 & \\
\hline$>0.62$ & $2 / 17(11.8)$ & $15 / 17(88.2)$ & 0.358 & \\
\hline Creatinine, mg/dL & $0.74(0.37-1.09)$ & $0.66(0.38-1.14)$ & 0.221 & \\
\hline$>0.72$ & $3 / 11(27 \cdot 3)$ & $8 / 11(72.7)$ & 0.269 & \\
\hline $\mathrm{eGFR}, \mathrm{mL} / \mathrm{min} / 1.73 \mathrm{~m}^{2}$ & $93.9(68.7-174.2)$ & $96.5(66.2-172.7)$ & 0.918 & \\
\hline$>93.5$ & $3 / 20(15 \cdot 0)$ & $17 / 20(85.0)$ & 0.519 & \\
\hline Baseline HCV RNA, $10^{3} \mathrm{IU} / \mathrm{mL}$ & $3,250(335-73,000)$ & $3,640(17-135,000)$ & 0.527 & \\
\hline$>881$ & $3 / 23(17.1)$ & $20 / 23(87.0)$ & 0.329 & 0.912 \\
\hline
\end{tabular}

Values are presented as number/total number (\%) or median (range).

SVR, sustained virological response; HCC, hepatocellular carcinoma; eGFR, estimated glomerular filtration rate; HCV, hepatitis C virus. 
Table 5. Clinical factors associated with rapid virological response

\begin{tabular}{|c|c|c|c|c|}
\hline \multirow{2}{*}{ Variable } & \multirow{2}{*}{ Patients without RVR } & \multirow{2}{*}{ Patients with RVR } & \multicolumn{2}{|c|}{$p$ value } \\
\hline & & & Univariate & Multivariate \\
\hline Total patients & $35 / 181(19 \cdot 3)$ & $146 / 181(80.7)$ & & \\
\hline Male sex & $13 / 76(17.1)$ & $63 / 76(82.9)$ & 0.326 & 0.454 \\
\hline Age, yr & $63(48-77)$ & $60(43-86)$ & 0.405 & \\
\hline$>60$ & $20 / 86(23.3)$ & $66 / 86(76.7)$ & 0.204 & \\
\hline Weight, kg & $58(45.7-93)$ & $61.4(35-104)$ & 0.685 & \\
\hline$>60$ & $15 / 92(16.3)$ & $77 / 92(83.7)$ & 0.294 & \\
\hline Liver cirrhosis & $14 / 50(28.0)$ & $36 / 50(72.0)$ & 0.056 & 0.026 \\
\hline Decompensated & o/6 (o) & $6 / 6(100)$ & 0.103 & \\
\hline History of HCC & $3 / 11(27.3)$ & $8 / 11(72.7)$ & 0.360 & 0.774 \\
\hline Diabetes & $3 / 30(10.0)$ & $27 / 30(90.0)$ & 0.119 & 0.245 \\
\hline Treatment naive & $23 / 143(16.1)$ & $120 / 143(83.9)$ & 0.031 & 0.067 \\
\hline Reduction of ribavirin dose & $4 / 32(12.5)$ & $28 / 32(87.5)$ & 0.206 & \\
\hline \multicolumn{5}{|l|}{ Laboratory findings } \\
\hline White blood cell count, / $\mu \mathrm{L}$ & $5,530(2,510-9,530)$ & $5,250(2,270-11,430)$ & 0.322 & \\
\hline$>5,280$ & $21 / 91(23.1)$ & $70 / 91(76.9)$ & 0.200 & \\
\hline Hemoglobin, g/dL & $13.3(9.9-17.1)$ & $13.4(9.5-17.7)$ & 0.545 & \\
\hline$>13.3$ & $16 / 90(17.8)$ & $74 / 90(82.2)$ & 0.597 & \\
\hline Platelets, $10^{3} / \mu \mathrm{L}$ & $172(46-329)$ & $179(23-425)$ & 0.154 & \\
\hline$>179$ & $16 / 89(18.0)$ & $73 / 89(82.0)$ & 0.649 & \\
\hline Aspartate transaminase, IU/L & $36(14-201)$ & $36(12-284)$ & 0.251 & \\
\hline$>36$ & $18 / 89(20.2)$ & $71 / 89(79.8)$ & 0.766 & \\
\hline Alanine aminotransferase, IU/L & $22(8-110)$ & $25(10-264)$ & 0.623 & \\
\hline$>25$ & $14 / 85(16.5)$ & $71 / 85(83.5)$ & 0.358 & \\
\hline Albumin, g/dL & $4.1(2.5-4.8)$ & $4.2(2.5-4.9)$ & 0.077 & 0.351 \\
\hline$>4.2$ & $13 / 86(15.1)$ & $73 / 86(84.9)$ & 0.171 & \\
\hline Total bilirubin, mg/dL & $0.62(0.2-2.1)$ & $0.62(0.23-2.52)$ & 0.750 & \\
\hline$>0.62$ & $17 / 89(19.1)$ & $72 / 89(80.9)$ & 0.937 & \\
\hline Creatinine, mg/dL & $0.66(0.37-1.14)$ & $0.74(0.42-1.71)$ & 0.012 & 0.015 \\
\hline$>0.72$ & $11 / 90(12.2)$ & $79 / 90(87.8)$ & 0.016 & \\
\hline $\begin{array}{l}\text { eGFR, } \mathrm{mL} / \mathrm{min} / 1.73 \mathrm{~m}^{2} \\
>93.5\end{array}$ & $\begin{array}{l}96.5(66.2-174.2) \\
20 / 90(22.2)\end{array}$ & $\begin{array}{l}92.7(32.6-165.6) \\
70 / 90(77.8)\end{array}$ & $\begin{array}{l}0.008 \\
0.346\end{array}$ & 0.795 \\
\hline Baseline HCV RNA, $10^{3} \mathrm{IU} / \mathrm{mL}$ & $3,640(171-135,000)$ & $742.5(0.24-72,750)$ & 0.004 & 0.027 \\
\hline$>881$ & $23 / 90(25.6)$ & $67 / 90(74.4)$ & 0.035 & \\
\hline
\end{tabular}

Values are presented as number/total number (\%) or median (range).

RVR, rapid virological response; HCC, hepatocellular carcinoma; eGFR, estimated glomerular filtration rate; HCV, hepatitis C virus.

reduced in 32 patients $(17.7 \%)$ due to anemia; these 32 patients received a mean $83.5 \%$ (range, $55 \%$ to $95 \%$ ) of their expected doses of RBV based on body weight. Reductions in RBV were not associated with RVR and SVR12 rates (Tables 3 and 5). Four patients did not complete RBV treatment, all due to economic problems. No AEs led to treatment discontinuation. Following the end of treatment, one patient was lost to follow-up. That pa- 
Table 6. Clinical factors associated with sustained virological response at 12 weeks in patients with a history of hepatocellular carcinoma

\begin{tabular}{|c|c|c|c|c|}
\hline \multirow{2}{*}{ Variable } & \multirow{2}{*}{$\begin{array}{l}\text { Patients without SVR12 } \\
\qquad(\mathrm{n}=3)\end{array}$} & \multirow{2}{*}{$\begin{array}{l}\text { Patients with SVR12 } \\
\qquad(\mathrm{n}=8)\end{array}$} & \multicolumn{2}{|c|}{$p$ value } \\
\hline & & & Univariate & Multivariate \\
\hline Male sex & $2(66.7)$ & $5(62.5)$ & 0.903 & \\
\hline Age, yr & $61(50-70)$ & $68(60-78)$ & 0.194 & 0.102 \\
\hline Weight, kg & $88.7(64.6-90.9)$ & $60.9(51.0-69.7)$ & 0.048 & 0.012 \\
\hline Liver cirrhosis & $2(66.7)$ & $8(100)$ & 0.102 & 0.087 \\
\hline Decompensated & o & $1(12.5)$ & 0.800 & \\
\hline Diabetes & $1(33 \cdot 3)$ & $2(25.0)$ & 0.792 & \\
\hline HCV treatment naive & $3(100)$ & $7(87.5)$ & 0.540 & \\
\hline HCC treatment before HCV treatment & & & 0.557 & \\
\hline Resection & 0 & $2(25)$ & & \\
\hline Radiofrequency ablation & $2(66.7)$ & $3(37.5)$ & & \\
\hline Transarterial chemoembolization & $1(33 \cdot 3)$ & $3(37 \cdot 5)$ & & \\
\hline HCC recurrence after HCV treatment & $2(66.7)$ & $4(50.0)$ & 0.637 & \\
\hline Reduction of ribavirin dose & 0 & $4(50.0)$ & 0.279 & \\
\hline \multicolumn{5}{|l|}{ Laboratory findings } \\
\hline White blood cell count, /uL & $3,260(2,910-3,640)$ & $4,445(3,120-6,550)$ & 0.048 & 0.051 \\
\hline Hemoglobin, g/dL & $13.3(11.6-13.6)$ & $13.1(9.9-14.7)$ & 1.000 & \\
\hline Platelets, $10^{3} / \mu \mathrm{L}$ & $61(48-98)$ & $141(59-189)$ & 0.133 & 0.085 \\
\hline Aspartate transaminase, IU/L & $82(68-115)$ & $33(25-60)$ & 0.012 & 0.006 \\
\hline Alanine aminotransferase, IU/L & $29(21-50)$ & $17(10-90)$ & 0.133 & 0.620 \\
\hline Albumin, g/dL & $3.8(2.5-4.2)$ & $3.8(3.1-4.4)$ & 0.630 & \\
\hline Total bilirubin, mg/dL & $0.76(0.70-.2 .1)$ & $0.83(0.28-1.49)$ & 0.776 & \\
\hline Creatinine, mg/dL & $0.79(0.64-1.09)$ & $0.75(0.46-0.96)$ & 1.000 & \\
\hline $\mathrm{eGFR}, \mathrm{mL} / \mathrm{min} / 1.73 \mathrm{~m}^{2}$ & $91.7(68.7-132.3)$ & $92.7(78.6-113.6)$ & 0.776 & \\
\hline Baseline HCV RNA, $10^{3} \mathrm{IU} / \mathrm{mL}$ & $409(332-788)$ & $322(25-3,340)$ & 0.776 & \\
\hline$\alpha$-Fetoprotein, $\mathrm{ng} / \mathrm{mL}$ & $45.5(29.4-86.9)$ & $5.5(1.2-22.5)$ & 0.012 & 0.008 \\
\hline Rapid virological response & $1(33 \cdot 3)$ & $2(25.0)$ & 0.792 & \\
\hline
\end{tabular}

Values are presented as number (\%) or median (range).

SVR, sustained virological response; HCV, hepatitis C virus; HCC, hepatocellular carcinoma; eGFR, estimated glomerular filtration rate.

tient died of pneumonia 2 months after the end of treatment. Because this patient had a history of bilobectomy due to lung cancer, death was regarded as independent of treatment for HCV.

\section{DISCUSSION}

This study analyzed the efficacy and safety of SOF and
RBV combination therapy in real-world patients infected with HCV GT2. IT'T analysis showed that the SVR12 rate was $92.8 \%$, slightly lower than that of a phase $3 \mathrm{~b}$ study in 129 Korean patients, which reported an SVR12 rate following SOF/RBV treatment for 12 weeks of approximately $97 \%$ [14]. The present study, however, included higher percentages of patients with LC (27.6\% vs. $10 \%)$ and those who had undergone previous treatment (21.0\% vs. $18.6 \%)$, as well as having a higher average age 
Table 7. Adverse events $(n=181)$

\begin{tabular}{|c|c|}
\hline Adverse event & No. (\%) \\
\hline \multicolumn{2}{|l|}{ Anemia } \\
\hline Grade $1 / 2$ & $31(17.1)$ \\
\hline Grade 3/4 & $1(0.6)$ \\
\hline \multicolumn{2}{|l|}{ Fatigue } \\
\hline Grade $1 / 2$ & $18(9.9)$ \\
\hline Grade 3/4 & 0 \\
\hline \multicolumn{2}{|l|}{ Nausea } \\
\hline Grade $1 / 2$ & $11(6.1)$ \\
\hline Grade 3/4 & o \\
\hline \multicolumn{2}{|c|}{ Urticaria or eruption } \\
\hline Grade 1/2 & $11(6.1)$ \\
\hline Grade 3/4 & 0 \\
\hline \multicolumn{2}{|l|}{ Headache } \\
\hline Grade 1/2 & $6(3.3)$ \\
\hline Grade 3/4 & o \\
\hline \multicolumn{2}{|l|}{ Diarrhea } \\
\hline Grade 1/2 & o \\
\hline Grade $3 / 4$ & $2(1.1)$ \\
\hline
\end{tabular}

(61.4 years vs. 55 years). Moreover, SVR12 rates in this study were lower than those in phase three studies from Japan (97\%) [15] and Taiwan (100\%) [16]. The percentages of treatment-experienced patients in these populations (41.1\% and 50.5\%, respectively) were higher than in our study, although average ages ( 57 and 53 years, respectively) and percentages of patients with LC (14\% and $15 \%$, respectively) were lower. Because the combination of older age and LC is associated with a lower SVR12 rate, our results demonstrated that SOF and RBV are effective in treating real-world patients infected with HCV GT2.

This study found that the achievement of RVR and no previous history of HCC were significant positive predictors of SVR12. Previous real-world studies found that low platelet count and esophageal varices were negative predictors of SVR [17], whereas absence of cirrhosis and higher serum albumin concentration were positive predictors of SVR12 [4]. Male sex has also been found to negatively predict SVR12 [18], but that study included patients infected with all genotypes of HCV. Moreover, these earlier studies did not evaluate RVR as a predictor of SVR12. To our knowledge, the present study is the first real world study in a Korean population to show that RVR was a positive predictor of SVR12 and to identify factors unrelated to LC that were predictive of SVR12 in patients infected with HCV GT2.

RVR was found to be an important positive predictor of SVR in patients treated with interferon, but not in patients treated with DAAs. RVR in patients treated with interferon was defined as the absence of detectable HCV RNA in blood using a sensitive assay with a lower limit of $50 \mathrm{IU} / \mathrm{mL}$ [19]. Recent advances in testing have enabled the measurement of viral loads as low as $15 \mathrm{IU} / \mathrm{mL}$. Moreover, these assays could differentiate between non-detectable HCV RNA and concentrations below the limit of detection $[20,21]$. One study involving 21,095 patients showed that SVR was dependent on the definition of RVR [22]. SVR rates in patients who achieved RVR were found to be $93.5 \%$ when RVR was defined as undetectable HCV RNA at 4 weeks, 91.8\% when RVR was defined as HCV RNA below the quantification limit, and 86.2\% when RVR was defined as HCV RNA $<42 \mathrm{IU} / \mathrm{mL}$. Because most patients in the POSITRON trial achieved RVR, defined as $<25 \mathrm{IU} / \mathrm{mL}$, this factor was not a predictor of SVR [23]. In the present study, RVR was defined as non-detection of HCV RNA at 4 weeks, with 28 of 34 patients who did not achieve RVR, including two of four patients with recurrence, having HCV RNA concentrations below the limit of quantitation. If RNA below the quantitative limit is regarded as RVR, then RVR in this study would not be predictive of SVR.

The risk factors for non-achievement of RVR were the presence of LC, high creatinine concentration, and high pre-treatment serum HCV RNA titer. Because many LC patients did not achieve RVR, we thought that their SVR12 rate would be low. LC is a risk factor for treatment failure, with guidelines recommending that these patients be treated with SOF/RBV for 16 weeks [12]. Treatment for 16 weeks was found to enhance the SVR12 rate of patients with LC, regardless of RVR, indicating that longer treatment could overcome the low SVR rate due to LC.

This study also found that the SVR12 rate in patients without LC was dependent on RVR, being significantly higher in those who did than did not achieve RVR (97.3\% vs. $81.0 \%, p=0.002$ ). Our findings suggest that treatment for 12 weeks may be insufficient for patients without LC who do not achieve RVR, whereas treatment for 16 weeks may improve their SVR12 rate. Indeed, we found 
that SVR12 rates did not differ significantly in patients with and without LC who did not achieve RVR (81.0\% vs. $85.7 \%, p=0.714)$. However, prolonged treatment is not always optimal, as it may increase the number of AEs associated with treatment. Therefore, additional studies may be required to determine whether prolonged treatment could enhance SVR12 rate in patients without LC who do not achieve RVR.

In this study, creatinine concentration and pre-treatment serum HCV RNA titer were risk factors for RVR, but not for SVR. Previous studies have reported that RNA titer did not affect RVR, perhaps due to the different definitions of RVR [23]. Furthermore, no previous study assessed the effect of creatinine concentration on RVR. Because SOF is predominantly excreted through the kidneys [24], guidelines advise that patients with low eGFR not be treated with this agent, as its serum concentrations may be too high. Conversely, SOF concentrations may be too low in patients with high eGFR, reducing RVR rates.

Another positive predictor of SVR12 in our study was the absence of a previous history of HCC. A study of 17,487 patients treated with DAAs found that the SVR rates in patients with and without a previous history of HCC were $74.5 \%$ and $91.9 \%$, respectively, indicating that HCC is a negative predictor of SVR [25]. SVR rates, however, did not differ significantly in HCV GT2-infected patients with and without a history of HCC. These findings suggest that HCC itself may be associated with lower SVR rates. Because HCV lives in HCC cells, the virus may be inaccessible to DAAs, reducing their antiviral effect [25]. In addition, HCCs develop in chronically inflamed livers, destroying their architecture, conditions that may alter hepatic immune responses and reduce responses to antiviral agents $[26,27]$. Because this study enrolled only patients without current HCC, the low SVR rate may be associated with the HCC environment, not HCC itself.

Multivariate analysis showed that high body weight, high aspartate transaminase concentration, and high $\alpha$-fetoprotein concentration were significantly associated with low SVR12 rate in patients with a history of HCC, suggesting that these factors may reflect certain conditions associated with HCC. Because the number of patients in this study with a history of HCC was too small, further studies are required to verify this hypothesis.
This study had several limitations. First, SOF with RBV is not the latest treatment regimen. However, this was the only treatment available through the National Health Insurance in Korea and the only option for patients with hepatic decompensation in some Asian countries. Second, the number of patients in each subgroup, such as those with HCC, was too small to investigate factors predictive of SVR in subgroups. Moreover, SVR12 could not be assessed in five patients. However, this study included patients at a single center, resulting in highly homogeneous data.

In conclusion, treatment with SOF/RBV was effective, safe, and tolerable in a real-world population of Korean patients infected with HCV GT2. RVR and no previous history of HCC were positive predictors of SVR12, while the presence of LC, high creatinine concentration, and high pre-treatment serum HCV RNA titer were negative predictors of RVR. Further studies are needed to verify factors predictive of lower SVR12 rate in patients with HCC.

\section{KEY MESSAGE}

1. In this study, rapid virological response (RVR) was defined as the absence of hepatitis $\mathrm{C}$ virus (HCV) RNA 4 weeks after starting sofosbuvir/ ribavirin.

2. RVR and no previous history of hepatocellular carcinoma were positive predictors of sustained virological response 12 weeks after the end of treatment (SVR12).

3. Presence of liver cirrhosis, high creatinine concentration, and high pre-treatment serum HCV RNA titer were negative predictors of RVR.

\section{Conflict of interest}

No potential conflict of interest relevant to this article was reported.

\section{Acknowledgments}

This work was supported by a 2-Year Research Grant of Pusan National University. 


\section{REFERENCES}

1. Gower E, Estes C, Blach S, Razavi-Shearer K, Razavi H. Global epidemiology and genotype distribution of the hepatitis C virus infection. J Hepatol 2014;61(1 Suppl):S45-S57.

2. Fattovich G, Stroffolini T, Zagni I, Donato F. Hepatocellular carcinoma in cirrhosis: incidence and risk factors. Gastroenterology 2004;127(5 Suppl 1):S35-S50.

3. Perz JF, Armstrong GL, Farrington LA, Hutin YJ, Bell BP. The contributions of hepatitis B virus and hepatitis $\mathrm{C}$ virus infections to cirrhosis and primary liver cancer worldwide. J Hepatol 2006;45:529-538.

4. Welzel TM, Nelson DR, Morelli G, et al. Effectiveness and safety of sofosbuvir plus ribavirin for the treatment of HCV genotype 2 infection: results of the real-world, clinical practice HCV-TARGET study. Gut 2017;66:1844-1852.

5. Ju W, Yang S, Feng S, et al. Hepatitis C virus genotype and subtype distribution in Chinese chronic hepatitis $\mathrm{C}$ patients: nationwide spread of HCV genotypes 3 and 6 . Virol J 2015;12:109.

6. Toyoda H, Kumada T, Takaguchi K, Shimada N, Tanaka $\mathrm{J}$. Changes in hepatitis $\mathrm{C}$ virus genotype distribution in Japan. Epidemiol Infect 2014;142:2624-2648.

7. Kim DY, Kim IH, Jeong SH, et al. A nationwide seroepidemiology of hepatitis C virus infection in South Korea. Liver Int 2013;33:586-594.

8. Shon HS, Choi HY, Kim JR, et al. Comparison and analysis of the prevalence of hepatitis $\mathrm{C}$ virus infection by region in the Republic of Korea during 2005-2012. Clin Mol Hepatol 2015;21:249-256.

9. van der Meer AJ, Veldt BJ, Feld JJ, et al. Association between sustained virological response and all-cause mortality among patients with chronic hepatitis $\mathrm{C}$ and advanced hepatic fibrosis. JAMA 2012;308:2584-2593.

10. Ioannou GN, Green PK, Berry K. HCV eradication induced by direct-acting antiviral agents reduces the risk of hepatocellular carcinoma. J Hepatol 2018;68:25-32.

11. AASLD/IDSA HCV Guidance Panel. Hepatitis C guidance: AASLD-IDSA recommendations for testing, managing, and treating adults infected with hepatitis $\mathrm{C}$ virus. Hepatology 2015;62:932-954.

12. Lawitz E, Mangia A, Wyles D, et al. Sofosbuvir for previously untreated chronic hepatitis C infection. N Engl J Med 2013;368:1878-1887.

13. European Association for the Study of the Liver. EASL recommendations on treatment of hepatitis C 2016. J
Hepatol 2017;66:153-194.

14. Ahn SH, Lim YS, Lee KS, et al. A phase 3b study of sofosbuvir plus ribavirin in treatment-naive and treatment-experienced Korean patients chronically infected with genotype 2 hepatitis C virus. J Viral Hepat 2016;23:358-365.

15. Omata M, Nishiguchi S, Ueno Y, et al. Sofosbuvir plus ribavirin in Japanese patients with chronic genotype 2 HCV infection: an open-label, phase 3 trial. J Viral Hepat 2014;21:762-768.

16. Kao JH, Chien RN, Chang TT, et al. A phase 3b study of sofosbuvir plus ribavirin in Taiwanese patients with chronic genotype 2 hepatitis $\mathrm{C}$ virus infection. Liver Int 2016;36:1101-1107.

17. Mangia A, Susser S, Piazzolla V, et al. Sofosbuvir and ribavirin for genotype $2 \mathrm{HCV}$ infected patients with cirrhosis: a real life experience. J Hepatol 2017;66:711-717.

18. Wu CJ, Roytman MM, Hong LK, et al. Real-world experience with sofosbuvir-based regimens for chronic hepatitis $\mathrm{C}$, including patients with factors previously associated with inferior treatment response. Hawaii J Med Public Health 2015;74(9 Suppl 2):3-7.

19. Yu JW, Wang GQ, Sun LJ, Li XG, Li SC. Predictive value of rapid virological response and early virological response on sustained virological response in HCV patients treated with pegylated interferon alpha-2a and ribavirin. $J$ Gastroenterol Hepatol 2007;22:832-836.

20. Sidharthan S, Kohli A, Sims Z, et al. Utility of hepatitis C viral load monitoring on direct-acting antiviral therapy. Clin Infect Dis 2015;60:1743-1751.

21. Laperche S, Bouchardeau F, Andre-Garnier E, et al. Interpretation of real-time PCR results for hepatitis $\mathrm{C}$ virus RNA when viral load is below quantification limits. J Clin Microbiol 2011;49:1113-1115.

22. Johnson K, Green PK, Ioannou GN. Implications of HCV RNA level at week 4 of direct antiviral treatments for hepatitis C. J Viral Hepat 2017;24:966-975.

23. Jacobson IM, Gordon SC, Kowdley KV, et al. Sofosbuvir for hepatitis $\mathrm{C}$ genotype 2 or 3 in patients without treatment options. N Engl J Med 2013;368:1867-1877.

24. Kirby BJ, Symonds WT, Kearney BP, Mathias AA. Pharmacokinetic, pharmacodynamic, and drug-interaction profile of the hepatitis $\mathrm{C}$ virus $\mathrm{NS}_{5} \mathrm{~B}$ polymerase inhibitor sofosbuvir. Clin Pharmacokinet 2015;54:677-690.

25. Beste LA, Green PK, Berry K, Kogut MJ, Allison SK, Ioannou GN. Effectiveness of hepatitis $C$ antiviral treatment in a USA cohort of veteran patients with hepatocellular 
carcinoma. J Hepatol 2017;67:32-39.

26. Wirth TC, Manns MP. The impact of the revolution in hepatitis C treatment on hepatocellular carcinoma. Ann Oncol 2016;27:1467-1474.
27. Hengst J, Schlaphoff V, Deterding K, et al. DAA-induced $\mathrm{HCV}$ clearance does not restore the altered cytokine and chemokine milieu in patients with chronic hepatitis C. J Hepatol 2016;64(2 Suppl):S417-S418. 
Han SY, et al. RVR and no HCC as predictors of SVR

Supplementary Table 1. Comparison of CP score between patients with HCC and those with LC but not HCC

\begin{tabular}{lccc}
\hline & HCC $(\mathrm{n}=11)$ & LC without HCC $(\mathrm{n}=40)$ & $p$ value \\
\hline CP score & $5.9 \pm 1.3$ & $5.4 \pm 0.9$ & 0.179 \\
CP A:B & $10: 1$ & $35: 5$ & 0.762 \\
SVR12 & $8 / 11(72.7)$ & $36 / 40(90)$ & 0.146 \\
\hline
\end{tabular}

Values are presented as mean \pm SD or number/total number $(\%)$.

CP, Child-Pugh; HCC, hepatocellular carcinoma; LC, liver cirrhosis; SVR, sustained virological response. 